\title{
On the Largest Part Size and Its Multiplicity of a Random Integer Partition
}

\author{
Ljuben Mutafchiev \\ American University in Bulgaria, 2700 Blagoevgrad, Bulgaria \\ and Institute of Mathematics and Informatics of the \\ Bulgarian Academy of Sciences \\ ljuben@aubg . edu
}

\begin{abstract}
Let $\lambda$ be a partition of the positive integer $n$ chosen uniformly at random among all such partitions. Let $L_{n}=L_{n}(\lambda)$ and $M_{n}=M_{n}(\lambda)$ be the largest part size and its multiplicity, respectively. For large $n$, we focus on a comparison between the partition statistics $L_{n}$ and $L_{n} M_{n}$. In terms of convergence in distribution, we show that they behave in the same way. However, it turns out that the expectation of $L_{n} M_{n}-L_{n}$ grows as fast as $\frac{1}{2} \log n$. We obtain a precise asymptotic expansion for this expectation and conclude with an open problem arising from this study.
\end{abstract}

Mathematics Subject Classifications: 05A17, 11P82, 60C05

Key words: integer partition, part size, multiplicity, expectation, asymptotic behavior

\section{Introduction and statement of the main re- sults}

Partitioning integers into summands (parts) is a subject of intensive research in combinatorics, number theory and statistical physics. If $n$ is a positive integer, then by a partition $\lambda$ of $n$, we mean the representation

$$
\lambda: \quad n=\lambda_{1}+\lambda_{2}+.+\lambda_{k}, \quad k \geq 1,
$$

where the positive integers $\lambda_{j}$ satisfy $\lambda_{1} \geq \lambda_{2} \geq \ldots \geq \lambda_{k}$. Let $\Lambda(n)$ be the set of all partitions of $n$ and let $p(n)=|\Lambda(n)|$ (by definition $p(0)=1$ regarding that the one partition of 0 is the empty partition). The number $p(n)$ is determined asymptotically by the famous partition formula of Hardy and Ramanujan [10]:

$$
p(n) \sim \frac{1}{4 n \sqrt{3}} \exp \left(\pi \sqrt{\frac{2 n}{3}}\right), \quad n \rightarrow \infty .
$$


A precise asymptotic expansion for $p(n)$ was found by Rademacher [13] (see also [2, Chapter 5]). Further on, for fixed integer $n \geq 1$, a partition $\lambda \in \Lambda(n)$ is selected uniformly at random. In other words, we assign the probability $1 / p(n)$ to each $\lambda \in \Lambda(n)$. We denote this probability measure on $\Lambda(n)$ by $\mathbb{P}$. Let $\mathbb{E}$ be the expectation with respect to $\mathbb{P}$. In this way, each numerical characteristic of $\lambda \in \Lambda(n)$ can be regarded as a random variable, or, a statistic produced by the random generation of partitions of $n$.

In this paper, we focus on two statistics of random integer partitions $\lambda \in$ $\Lambda(n): L_{n}=L_{n}(\lambda)=\lambda_{1}$, which is the largest part size in representation (1) and $M_{n}=M_{n}(\lambda)$, equal to the multiplicity of the largest part $\lambda_{1}$ (i.e., $M_{n}(\lambda)=$ $m, 1 \leq m \leq k-1$, if $\lambda_{1}=\ldots=\lambda_{m}>\lambda_{m+1} \geq \ldots \geq \lambda_{k}$ in (1), and $M_{n}(\lambda)=k$, if $\left.\lambda_{1}=\ldots=\lambda_{k}\right)$.

Each partition $\lambda \in \Lambda(n)$ has a unique graphical representation called Ferrers diagram [2, Chapter 1]. It illustrates (1) by the two-dimensional array of dots, composed by $\lambda_{1}$ dots in the first (most left) row, $\lambda_{2}$ dots in the second row,..., and so on, $\lambda_{k}$ dots in the $k$ th row. Therefore, a Ferrers diagram may be considered as a union of disjoint blocks (rectangles) of dots whose side lengths represent the part sizes and their multiplicities of the partition $\lambda$, respectively. For instance Figure 1 illustrates the partition $7+5+5+5+4+2+1+1+1$ of 31 in which $L_{n}=7$ and $M_{n}=1$.

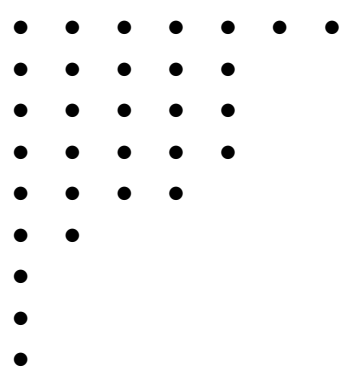

Figure 1

The earliest asymptotic results on random integer partition statistics has been obtained long ago by Husimi [11] and Erdös and Lehner [5]. Husimi has derived an asymptotic expansion for $\mathbb{E}\left(L_{n}\right)$ in the context of a statistical physics model of a Bose gas. Erdös and Lehner were apparently the first who have studied random partition statistics in terms of probabilistic limit theorems. In fact, they showed that

$$
\lim _{n \rightarrow \infty} \mathbb{P}\left(\frac{L_{n}}{\sqrt{n}}-\frac{1}{2 c} \log n \leq u\right)=H(u),
$$

where

$$
H(u)=\exp \left(-\frac{1}{c} e^{-c u}\right), \quad-\infty<u<\infty
$$

and

$$
c=\frac{\pi}{\sqrt{6}} .
$$


Husimi's asymptotic result was subsequently reconfirmed by Kessler and Livingston [12. Higher moments of $L_{n}$ were studied in [14. A general method providing asymptotic expansions of expectations of integer partition statistics was recently proposed by Grabner et al. 9]. Among the numerous examples, they derived a rather complete asymptotic expansion for $\mathbb{E}\left(L_{n}\right)$, namely,

$$
\begin{aligned}
& \mathbb{E}\left(L_{n}\right)=\frac{\sqrt{n}}{2 c}(\log n+2 \gamma-2 \log c)+\frac{\log n}{2 c^{2}}+\frac{1}{4} \\
& +\frac{1+2 \gamma-2 \log c}{4 c^{2}}+O\left(\frac{\log n}{n}\right), \quad n \rightarrow \infty,
\end{aligned}
$$

where $c$ is given by (4) and $\gamma=0.5772 \ldots$ denotes the Euler's constant (see 9 , Proposition 4.2]). Notice that by conjunction of the Ferrers diagram the largest part and the total number of parts in a random partition of $n$ are identically distributed for any $n$. The sequence $\left\{p(n) \mathbb{E}\left(L_{n}\right)\right\}_{n \geq 1}$ is given in [15] as A006128.

There are serious reasons to believe that the multiplicity $M_{n}$ of the largest part of a random partition of $n$ behaves asymptotically in a much simpler way than many other partition statistics. Grabner and Knopfmacher 8 used ErdösLehner limit theorem (2) to establish that

$$
\lim _{n \rightarrow \infty} \mathbb{E}\left(M_{n}\right)=1 .
$$

In addition, among many other important asymptotic results, Fristedt, in his remarkable paper [7], showed that, with probability tending to 1 as $n \rightarrow \infty$, the first $m_{n}$ largest parts in a random partition of $n$ are distinct if $m_{n}=o\left(n^{1 / 4}\right)$. Hence it may not be that $L_{n}$ constitutes the main contribution to $n$ by a single part size and some smaller part sizes may occur with sufficient multiplicity so that the products of these part sizes with their multiplicities could be much larger than $L_{n}$. In terms of the Ferrers diagram this means that its first block has typically smaller area than several next block areas with larger heights (multiplicities of parts).

Our aim in this paper is to study the asymptotic behavior of the area $L_{n} M_{n}$ of the first block in the Ferrers diagram of a random partition of $n$. We show some similarities and differences between the single part size $L_{n}$ and its corresponding block area $L_{n} M_{n}$. As a first step, we obtain a distributional result for $M_{n}$ that confirms the limit in (6).

Theorem 1 For any $n \geq 1$, we have

$$
\mathbb{P}\left(M_{n}=1\right)=\frac{p(n-1)}{p(n)} .
$$

In addition, if $n \rightarrow \infty$, then

$$
\mathbb{P}\left(M_{n}=1\right)=1-\frac{c}{\sqrt{n}}+\frac{1+c^{2} / 2}{n}+O\left(n^{-3 / 2}\right),
$$

where the constant $c$ is given by (4). 
Combining the Erdös-Lehner limit theorem (2) with (8), one can easily observe that the limiting distributions of $L_{n}$ and $L_{n} M_{n}$ coincide under the same normalization.

Corollary 1 For any real $u$, we have

$$
\lim _{n \rightarrow \infty} \mathbb{P}\left(\frac{L_{n} M_{n}}{\sqrt{n}}-\frac{1}{2 c} \log n \leq u\right)=H(u),
$$

where $H(u)$ and $c$ are given by (3) and (4), respectively.

Although $L_{n}$ and $L_{n} M_{n}$ follow the same limiting distribution, the difference in means $\mathbb{E}\left(L_{n} M_{n}\right)-\mathbb{E}\left(L_{n}\right)$ grows as fast as $\frac{1}{2} \log n$. A complete estimate is given in the following

Theorem 2 If $n \rightarrow \infty$, then, as $n \rightarrow \infty$,

$$
\mathbb{E}\left(L_{n} M_{n}\right)=\mathbb{E}\left(L_{n}\right)+\frac{1}{2} \log n-C+O(1 / \log n),
$$

where $C=\log c+1-\gamma=0.67165 \ldots$ and $\mathbb{E}\left(L_{n}\right)$ and $c$ are given by (5) and (4), respectively.

Remark 1. The sequence $\left\{p(n) \mathbb{E}\left(L_{n} M_{n}\right)\right\}_{n \geq 1}$ is given as A092321 in 15.

The proofs of Theorems 1 and 2 are based on generating function identities established in [3] that involve products of the form $P(x) G(x)$, where $P(x)$ is the Euler partition generating function

$$
P(x):=\sum_{n=0}^{\infty} p(n) x^{n}=\prod_{j=1}^{\infty}\left(1-x^{j}\right)^{-1}
$$

and $G(x)$ is a function which is analytic in the open unit disk and does not grow too fast as $x \rightarrow 1$. Our asymptotic expansions in (8) and Theorem 2 are obtained using a general asymptotic result of Grabner et al. 9 for the $n$th coefficient $x^{n}[P(x) G(x)]$. In the proof of Theorem 2 we also apply a classical way of estimating the growth of a power series around its main singularity.

We organize the paper as follows. Section 2 contains some auxiliary facts related to generating functions and the asymptotic analysis of their coefficients. In Section 3 we present the proofs of the main results. Finally. in Section 4 we conclude with an open problem on the position of $L_{n} M_{n}$ in the sequence of ordered block areas of a random Ferrers diagram.

\section{Preliminaries: Generating Functions and an Asymptotic Scheme}

We start with two generating function identities. In the next two lemmas $P(x)$ is the generating function given by (9) and by definition $\prod_{1}^{0}:=1$. 
Lemma 1 (i) For any positive integer $m$, we have

$$
\sum_{n=1}^{\infty} p(n) \mathbb{P}\left(M_{n}=m\right) x^{n}=x^{m} \prod_{j \geq m}\left(1-x^{j}\right)^{-1}=P(x) x^{m} \prod_{j=1}^{m-1}\left(1-x^{j}\right) .
$$

(ii) We have

$$
\sum_{n=1}^{\infty} p(n) \mathbb{E}\left(L_{n} M_{n}\right) x^{n}=\sum_{k=1}^{\infty} \frac{k x^{k}}{1-x^{k}} \prod_{j=1}^{k}\left(1-x^{j}\right)^{-1}=P(x) F(x),
$$

where

$$
F(x)=\sum_{k=1}^{\infty} \frac{k x^{k}}{1-x^{k}} \prod_{j=k+1}^{\infty}\left(1-x^{j}\right) .
$$

Sketch of the proof. Part (i) is the last conclusion of Theorem 2.3 from 3 . Part (ii) is given in A092321 of [15]. It also follows from Proposition 4.1 in [3].

We shall essentially use the main result from [9, Theorem 2.3]. We present here only slight modifications of those parts of this theorem that we will need for our further asymptotic analysis. Furthermore, by $\log x$ we denote the main branch of the logarithmic function that satisfies the inequality $\log x<0$ if $0<x<1$.

Lemma 2 Suppose that, for some constants $K>0$ and $\eta<1$, the function $G(x)$ satisfies

$$
G(x)=O\left(e^{K /(1-|x|)^{\eta}}\right), \quad|x| \rightarrow 1 .
$$

(i) Let $G\left(e^{-t}\right)=a t^{b}+O(|f(t)|)$ as $t \rightarrow 0$, $⿻$ t $>0$, where $b \geq 0$ is an integer and $a$ is real number. Then, we have

$$
\begin{aligned}
& \frac{1}{p(n)} x^{n}[P(x) G(x)]=a\left(\frac{2 \pi}{\sqrt{24 n-1}}\right)^{b} \frac{s}{s-1} \sum_{j=0}^{b+1} \frac{(b+j+1) !}{j !(b+j-1) !}\left(-\frac{1}{2 s}\right)^{j} \\
& +O\left(e^{-2 s}\right)+O\left(e^{-n^{1 / 2-\epsilon}}+f\left(c / \sqrt{n}+O\left(n^{-1 / 2-\epsilon}\right)\right)\right.
\end{aligned}
$$

for any $\epsilon \in(0,(1-\eta) / 2)$, where

$$
s=\sqrt{\frac{2 \pi^{2}}{s}\left(n-\frac{1}{24}\right)}=2 c \sqrt{n-\frac{1}{24}}
$$

and $c$ is given by (4).

(ii) Suppose that $G(x)$ satisfies condition (11) and, for $t=u+i v$, let $G\left(e^{-t}\right)=a \log \frac{1}{t}+O(f(|t|))$ as $t \rightarrow 0$, where $u>0, v=O\left(u^{1+\epsilon}\right)$ as $u \rightarrow 0^{+}$, where $\epsilon$ and $a$ are as in part (i). Then, we have

$$
\frac{1}{p(n)} x^{n}[P(x) G(x)]=a \log \left(\frac{\sqrt{24 n-1}}{2 \pi}\right)+O\left(n^{-1 / 2}+f\left(c / \sqrt{n}+O\left(n^{-1 / 2-\epsilon}\right)\right)\right.
$$

with c given by (4). 
As in [9], we remark that parts (i) and (ii) can be combined so that Lemma 2 generalizes to mixed asymptotic expansions involving sums of powers of $t$ and logarithms of $1 / t$. The proof of Lemma 2, based on the saddle point method, is presented in [9, Section 3].

\section{Proofs}

\section{Proof of Theorem 1.}

First, we set $m=1$ in Lemma 1(i). We have

$$
\sum_{n=1}^{\infty} \mathbb{P}\left(M_{n}=1\right) x^{n}=x P(x)
$$

This implies at once (7). The asymptotic behavior of the quotient in (7) may be found using Rademacher's "exact-asymptotic" formula [13] (see also [2, Chapter 5]). It seems that a quicker way is to apply the result of Lemma 2(i). Here we have $G(x)=x$, which obviously satisfies (11). Setting $x=e^{-t}$ and expanding $e^{-t}$ by Taylor formula, we can write there as many powers of $t$ as we wish, which will be transferred into powers of $n^{-1 / 2}$ in the asymptotic expansion of $\mathbb{P}\left(M_{n}=1\right)$. We decide to bound the error of estimation up to a term of order $O\left(n^{-3 / 2}\right)$ and write

$$
e^{-t}=1-t+\frac{1}{2} t^{2}+f(t)
$$

with

$$
f(t)=\sum_{j=3}^{\infty} \frac{t^{j}}{j !}
$$

The representation (14) requires to apply Lemma 2(i) twice: for the term $-t$ with $a=-1$ and $b=1$ and for the term $\frac{1}{2} t^{2}$ with $a=1 / 2$ and $b=2$. Furthermore, (15) implies that $f\left(c / \sqrt{n}+O\left(n^{-1 / 2}-\epsilon\right)\right)=O\left(n^{-3 / 2}\right)$. Thus, from (13) it follows that

$$
\mathbb{P}\left(M_{n}=1\right)=\frac{x^{n}[x P(x)]}{p(n)}=1-A_{1}(n)+A_{2}(n)+O\left(n^{-3 / 2}\right) .
$$

The computation of $A_{1}(n)$ and $A_{2}(n)$ is based on Lemma 2(i) with $s$ given by (12). We have

$$
\begin{aligned}
& A_{1}(n)=\frac{2 \pi}{\sqrt{24 n-1}} \frac{s}{s-1} \sum_{j=0}^{2} \frac{(2+j) !}{j !(2-j) !}\left(-\frac{1}{2 s}\right)^{j} \\
& =\frac{c}{\sqrt{n}}\left(1-\frac{1}{24 n}\right)^{-1 / 2}\left(1-\frac{2}{s}+\frac{1}{s^{2}}+O\left(s^{-3}\right) O\left(e^{-2 s}\right)\right. \\
& =\frac{c}{\sqrt{n}}\left(1+\frac{1}{48 n}+O\left(n^{-2}\right)\right)\left(1-\frac{1}{c \sqrt{n}}\left(1-\frac{1}{24 n}\right)^{-1 / 2}+O\left(n^{-1}\right)\right)(17) \\
& =-\frac{c}{\sqrt{n}}\left(1+O\left(n^{-1}\right)\right)\left(1-\frac{1}{c \sqrt{n}}+O\left(n^{-1}\right)\right)=-\frac{c}{\sqrt{n}}-\frac{1}{n}+O\left(n^{-3 / 2}\right) .
\end{aligned}
$$


In the same way, for $A_{2}(n)$, we obtain

$$
A_{2}(n)=\frac{c^{2}}{2 n}+O\left(n^{-3 / 2}\right) .
$$

The proof is completed by substituting (17) and (18) into (16).

Proof of the Corollary.

The total probability formula and the asymptotic estimate given in Theorem 1 imply that

$$
\begin{aligned}
& \mathbb{P}\left(\frac{L_{n} M_{n}}{\sqrt{n}}-\frac{1}{2 c} \log n \leq u\right)=\mathbb{P}\left(\frac{L_{n} M_{n}}{\sqrt{n}}-\frac{1}{2 c} \log n \leq u \mid M_{n}=1\right) \mathbb{P}\left(M_{n}=1\right) \\
& +\mathbb{P}\left(\frac{L_{n} M_{n}}{\sqrt{n}}-\frac{1}{2 c} \log n \leq u \mid M_{n} \neq 1\right) \mathbb{P}\left(M_{n} \neq 1\right) \\
& =\mathbb{P}\left(\frac{L_{n}}{\sqrt{n}}-\frac{1}{2} \log n \leq u\right)(1+O(1 / \sqrt{n}))+O(1 / \sqrt{n}) .
\end{aligned}
$$

Hence the Corollary follows easily from Erdös and Lehner's result (2).

\section{Proof of Theorem 2.}

First, we represent the function $F(x)$ given by (10) as

$$
F(x)=F_{1}(x)+F_{2}(x),
$$

where

$$
\begin{aligned}
& F_{1}(x)=\sum_{k=1}^{\infty} k x^{k} \prod_{j=k+1}^{\infty}\left(1-x^{j}\right), \\
& F_{2}(x)=\sum_{k=1}^{\infty} k\left(\sum_{l=2}^{\infty} x^{k l}\right) \prod_{j=k+1}^{\infty}\left(1-x^{j}\right) \\
& =\sum_{k=1}^{\infty} \frac{k x^{2 k}}{1-x^{k}} \prod_{j=k+1}^{\infty}\left(1-x^{j}\right) .
\end{aligned}
$$

Grabner and Knopfmacher [8, formula 6.2] found a simple alternative representation for $F_{1}(x)$ :

$$
F_{1}(x)=\sum_{k=1}^{\infty} \frac{x^{k}}{1-x^{k}}
$$

It is also known that

$$
\sum_{n=1}^{\infty} p(n) \mathbb{E}\left(L_{n}\right) x^{n}=P(x) F_{1}(x)
$$

(see, e.g., [9, p. 1059]). In addition, Grabner et al. [9, p. 1084] used Mellin trasform technique to show that

$$
F_{1}\left(e^{-t}\right)=\frac{\log (1 / t)+\gamma}{t}+\frac{1}{4}-\frac{t}{144}+O\left(|t|^{3}\right), \quad t \rightarrow 0 .
$$


From this expansion and their main result (see also both parts of Lemma 2) they derived asymptotic formula (5) for $\mathbb{E}\left(L_{n}\right)$. From (19) it follows that $x^{n}[F(x)]=$ $x^{n}\left[F_{1}(x)\right]+x^{n}\left[F_{2}(x)\right]$, which in turn implies that

$$
\mathbb{E}\left(L_{n} M_{n}\right)=\mathbb{E}\left(L_{n}\right)+\frac{x^{n}\left[F_{2}(x)\right]}{p(n)} .
$$

The asymptotic analysis of the second summand in the right-hand side of (22) will be based on Lemma 2(ii). So, we need a suitable expansion for $F_{2}\left(e^{-t}\right)$. We set $t=u+i v$ with $u$ and $v$ satisfying the conditions of Lemma 2(ii) and focus on an asymptotic estimate for $F_{2}\left(e^{-u}\right)$ as $u \rightarrow 0^{+}$. For the sake of convenience, we also set

$$
g_{k}(u)=\prod_{j=k+1}^{\infty}\left(1-e^{-j u}\right), \quad u>0 .
$$

Representing $g_{k}(u)$ as a Riemann sum with step size $u \rightarrow 0^{+}$and replacing it by the corresponding integral, we obtain the following basic estimate:

$$
g_{k}(u)=\exp \left(\sum_{j>k} \log \left(1-e^{-j u}\right)\right)=\exp \left(\frac{1}{u} \int_{k u}^{\infty} \log \left(1-e^{-w}\right) d w+o(1)\right) .
$$

Next, we proceed with the representation:

$$
F_{2}\left(e^{-u}\right)=\sum_{j=1}^{4} S_{j}(u)
$$

where by (21) and (23)

$$
S_{j}(u)=\sum_{k \in I_{j}} \frac{k e^{-2 k u}}{1-e^{-k u}} g_{k}(u), \quad j=1,2,3,4 .
$$

The subintervals $I_{j}$ are defined as follows. Let $\alpha>0$ be fixed. We set

$$
\begin{gathered}
I_{1}=[1, \alpha / u] \\
I_{2}=\left(\frac{\alpha}{u}, \frac{1}{u}\left(\log \frac{1}{u}-\log \log \left(\frac{1}{u}\right)^{3}\right)\right] \\
I_{3} \\
=\left(\frac{1}{u}\left(\log \frac{1}{u}-\log \log \frac{1}{u}-\log 3\right), \frac{1}{u}\left(\log \frac{1}{u}+\log \log \frac{1}{u}+\log 2\right)\right], \\
I_{4}=\left(\frac{1}{u}\left(\log \frac{1}{u}+\log \log \left(\frac{1}{u}\right)\right)+\log 2, \infty\right) .
\end{gathered}
$$

We start with an estimate for $S_{1}(u)$. Since, for $k \in I_{1}$,

$$
\int_{k u}^{\infty} \log \left(1-e^{-w}\right) d w \leq \int_{\alpha}^{\infty} \log \left(1-e^{-w}\right) d w=-c_{\alpha}<0
$$


from (24) we get

$$
g_{k}(u)=O\left(e^{-c_{\alpha} / u}\right) .
$$

Hence, using (27), we obtain

$$
\begin{aligned}
& S_{1}(u)=O\left(e^{-c_{\alpha} / u}\right) \sum_{u \leq k u \leq \alpha} \frac{k e^{-2 k u}}{1-e^{-k u}} \\
& =O\left(u^{-2} e^{-c_{\alpha} / u}\right) \int_{0}^{\alpha} \frac{w e^{-2 w}}{1-e^{-w}} d w=O\left(u^{-2} e^{-c_{\alpha} / u}\right) .
\end{aligned}
$$

As a preparation for the estimate of $S_{2}(u)$, we first note that a single integration by parts in the integral from the right-hand side of (24) yields

$$
\int_{k u}^{\infty} \log \left(1-e^{-w}\right) d w=-k u \log \left(1-e^{-k u}\right)-\int_{k u}^{\infty} \frac{w}{e^{w}-1} d w .
$$

On the other hand, from formula 27.1.2 in [1] we have

$$
\begin{aligned}
& \int_{y}^{\infty} \frac{w}{e^{w}-1} d w=y \sum_{k=1}^{\infty} \frac{e^{-k y}}{k}+\sum_{k=1}^{\infty} \frac{e^{-k y}}{k^{2}} \\
& =-y \log \left(1-e^{-y}\right)+e^{-y}+O\left(e^{-2 y}\right), \quad y \rightarrow \infty .
\end{aligned}
$$

Applying this estimate to (24), we obtain

$$
g_{k}(u)=\exp \left(-\frac{1}{u} e^{-k u}+O\left(u^{-1} e^{-2 k u}\right)+o(1)\right)
$$

for all $k$ that satisfy $k u \rightarrow \infty$ as $u \rightarrow 0^{+}$. So, from (28) it follows that

$$
\begin{aligned}
& S_{2}(u)=\frac{1+o(1)}{u} \sum_{k \in I_{2}} \frac{k u e^{-2 k u}}{1-e^{-k u}} \exp \left(-\frac{1}{u} e^{-k u}+O\left(u^{-2} e^{-2 k u}\right)\right) \\
& =O\left(\frac{\log \frac{1}{u}}{u}\left(\sum_{k \in I_{2}} \frac{e^{-2 \alpha}}{1-e^{-\alpha}}\right) \exp \left(-\log \left(\frac{1}{u}\right)^{3}+O\left(\frac{1}{u} e^{-2 \log \frac{1}{u}}\right)\right)\right) \\
& =O\left(\frac{u^{3} \log ^{2} \frac{1}{u}}{u^{2}}\right)=O\left(u \log ^{2} \frac{1}{u}\right), \quad u \rightarrow 0^{+} .
\end{aligned}
$$

(In the third line of (33) we have also used that the sum over $I_{2}$ contains at most $\frac{1}{u} \log \frac{1}{u}$ summands.)

We proceed now to the estimate for $S_{3}(u)$, whose contribution to $F_{2}\left(e^{-u}\right)$ is the most essential one. First, it is easy to observe that

$$
0 \leq \frac{1}{1-e^{-k u}}=O\left(u \log \frac{1}{u}\right), \quad u \rightarrow 0^{+}
$$


uniformly for all $k \in I_{3}$. Hence, approximating once again a Riemann sum by the corresponding integral, we obtain

$$
\begin{aligned}
& S_{3}(u)=\left(1+O\left(u \log \frac{1}{u}\right)\right) u^{-2} \int_{\log \frac{1}{u}-\log \log \frac{1}{u}-\log 3}^{\log +\log \log \frac{1}{u}+\log 2} w e^{-2 w} e^{-\frac{1}{u} e^{-w}} d w \\
& =\left(1+O\left(u \log \frac{1}{u}\right)\right) \int_{-\log \log \frac{1}{u}-\log 3}^{\log \log \frac{1}{u}+\log 2}\left(z+\log \frac{1}{u}\right) e^{-2 z} e^{-e^{-z}} d z .
\end{aligned}
$$

Changing the variable in (34), we represent $S_{3}(u)$ as follows:

$$
S_{3}(u)=\left(J_{1}(u)+J_{2}(u)\right)\left(1+O\left(u \log \frac{1}{u}\right)\right),
$$

where

$$
\begin{aligned}
& J_{1}(u)=\int_{2 / \log \frac{1}{u}}^{3 \log \frac{1}{u}}(-\log y) y e^{-y} d y, \\
& J_{2}(u)=\left(\log \frac{1}{u}\right) \int_{2 / \log \frac{1}{u}}^{3 \log \frac{1}{u}} y e^{-y} d y .
\end{aligned}
$$

An easy calculation based on an asymptotic estimate for the incomplete gamma function [1, formula 6.5.32] shows that

$$
\begin{aligned}
& \int_{2 / \log \frac{1}{u}}^{3 \log \frac{1}{u}} y e^{-y} d y=\int_{0}^{\infty} y e^{-y} d y-\int_{0}^{2 / \log \frac{1}{u}} y e^{-y} d y-\int_{3 \log \frac{1}{u}}^{\infty} y e^{-y} d y \\
& =1-O\left(\left(\log \frac{1}{u}\right)^{-2}\right)-O\left(\left(\log \frac{1}{u}\right) e^{-3 \log \frac{1}{u}}\right)=1-O\left(\left(\log \frac{1}{u}\right)^{-2}\right) .
\end{aligned}
$$

Inserting this estimate into (37), we obtain

$$
J_{2}(u)=\log \frac{1}{u}+O\left(1 / \log \frac{1}{u}\right) .
$$

For the estimate of $J_{1}(u)$, we recall that

$$
\int_{0}^{\infty} y e^{-y}(\log y) d y=1-\gamma
$$

(see, e.g., 44, formula 865.902]. Hence we have

$$
J_{1}(u)=\gamma-1-\int_{0}^{2 / \log \frac{1}{u}} y e^{-y}(-\log y) d y-\int_{3 \log \frac{1}{u}}^{\infty} y e^{-y}(-\log y) d y .
$$

Both integrals in the right-hand side of this representation are negligible. The first one can be estimated bounding $e^{-y}$ by 1 and then integrating by parts. This gives a bound of order $O\left(\log \log \frac{1}{u} / \log ^{2} \frac{1}{u}\right)$. For the second one we can 
use once again [1, formula 6.5.32], which leads to the upper bound $O\left(u^{3} \log ^{2} \frac{1}{u}\right)$. Thus we have

$$
J_{1}(u)=\gamma-1+O\left(\left(\log \log \frac{1}{u}\right) / \log ^{2} \frac{1}{u}\right) .
$$

Combining (35) - (39), we finally obtain

$$
S_{3}(u)=\log \frac{1}{u}+\gamma-1+O\left(\frac{1}{\log \frac{1}{u}}\right) .
$$

We end up our analysis with an estimate for $S_{4}(u)$. First, we bound the last exponent in the right-hand side of (32) by 1 and then, as previously, we approximate $S_{4}(u)$ by an integral, which can be bounded using again [1, formula 6.5.32]. Then, in a similar way, from (30) we obtain

$$
\begin{aligned}
& S_{4}(u)=O\left(u^{-2} \int_{\log \frac{1}{u}+\log \log \frac{1}{u}+\log 2}^{\infty} w e^{-2 w} d w\right) \\
& =O\left(\int_{\log \log \frac{1}{u}+\log 2}^{\infty}\left(z+\log \frac{1}{u}\right) e^{-2 z} d z\right) \\
& =O\left(\left(\log \log \frac{1}{u}\right) / \log ^{2} \frac{1}{u}\right)+O\left(1 / \log \frac{1}{u}\right)=O\left(1 / \log \frac{1}{u}\right) .
\end{aligned}
$$

Now, from (25), (31), (33), (40) and (41) it follows that

$$
\begin{aligned}
& F_{2}\left(e^{-u}\right)=O\left(u^{-2} e^{-c_{\alpha} / u}\right)+O\left(u^{2} \log \frac{1}{u}\right)+\log \frac{1}{u}+\gamma-1+O\left(1 / \log \frac{1}{u}\right) \\
& =\log \frac{1}{u}+\gamma-1+O\left(1 / \log \frac{1}{u}\right) .
\end{aligned}
$$

To transfer the variable $u$ in (42) into $t=u+i v$, we recall the relationship between $u$ and $v$ in Lemma 2(ii). First, we note that

$$
\log \frac{1}{t}=\log \frac{1}{u}+\log \frac{1}{1+i v / u}=\log \frac{1}{u}+O(|v| / u)=\log \frac{1}{u}+O\left(u^{\epsilon}\right)
$$

and

$$
e^{-t}=e^{-u} e^{-i v}=e^{-u}\left(1+O(|v|)=e^{-u}+O\left(u^{1+\epsilon}\right)\right.
$$

as $u \rightarrow 0^{+}$. Hence, by Taylor formula,

$$
F_{2}\left(e^{-t}\right)=F_{2}\left(e^{-u}\right)+O\left(u^{1+\epsilon}\left|\frac{d}{d u} F_{2}\left(e^{-u}\right)\right|\right) .
$$

The last error term can be estimated following the same line of reasoning. We outline briefly the proof. By (21) and (23) we have

$$
\begin{aligned}
& \frac{d}{d u} F_{2}\left(e^{-u}\right)=-\sum_{k=1}^{\infty} \frac{k^{2} e^{-k u}}{1-e^{-k u}} g_{k}(u)-\sum_{k=1}^{\infty} \frac{k^{2} e^{-k u}}{\left(1-e^{-k u}\right)^{2}} g_{k}(u) \\
& -\sum_{k=1}^{\infty} \frac{k e^{-k u}}{1-e^{-k u}} g_{k}^{\prime}(u),
\end{aligned}
$$


where

$$
g_{k}^{\prime}(u)=g_{k}(u) \sum_{j=k+1}^{\infty} \frac{j e^{-j u}}{1-e^{-j u}} .
$$

The main contribution to the asymptotic of $\left|\frac{d}{d u} F_{2}\left(e^{-u}\right)\right|$ as $u \rightarrow 0^{+}$is given by the first term of the right-hand side of (46) (since it contains the factor $k^{2}$ ). If we break up the range of summation as previously into the union $\bigcup_{j=1}^{4} I_{j}$ (see (27) - (30) ), we can again conclude that the contribution of the sum over $I_{3}$ is the largest one. An approximation by a Riemann integral requires division and multiplication by the cube of the step size, $u^{3}$. Hence, instead of $u^{-2}$, the factor $u^{-3}$ will multiply the same integral from the right-hand side of (34). An argument similar to (40) and (42) implies the following bound:

$$
\frac{d}{d u} F_{2}\left(e^{-u}\right)=O\left(\frac{1}{u} \log \frac{1}{u}\right), \quad u \rightarrow 0^{+} .
$$

Consequently, the remainder term in (45) is $O\left(u^{\epsilon} \log \frac{1}{u}\right)$. Combining this with (42) - (46), we obtain

$$
F_{3}\left(e^{-t}\right)=\log \frac{1}{t}+\gamma-1+O\left(1 / \log \frac{1}{t}\right), \quad t \rightarrow 0 .
$$

Therefore, we are ready to apply Lemma 2(ii) with $G(x):=F_{2}(x)$ and $f(t)=$ $1 / \log \frac{1}{t}$. We obtain

$$
\frac{1}{p(n)} x^{n}\left[F_{2}(x)\right]=\log \frac{c}{\sqrt{n}}+\gamma-1+O(1 / \log n), \quad n \rightarrow \infty .
$$

Now, we recall (22), which in combination with (47) completes the proof of Theorem 2.

\section{Concluding Remarks}

The main goal of this study is the comparison between the typical growths of the first block area $L_{n} M_{n}$ and its base length $L_{n}$ in the Ferrers diagram of a random integer partition $n$. It turns out that the leading terms in the asymptotic expansions of the expectations of these two statistics are the same for large $n$; both are equal to $\frac{\sqrt{n}}{2 c} \log n$. Erdös and Lehner's limit theorem (2) and Theorem 1 show that this leading term controls the weak convergence of $L_{n}$ and $L_{n} M_{n}$ both tending to a Gumbel distributed random variable after one and the same appropriate normalization. The expectations of $L_{n}$ and $L_{n} M_{n}$ are, however, different for large $n$. In fact, by Theorem 2 , that

$$
\lim _{n \rightarrow \infty}\left(\mathbb{E}\left(L_{n} M_{n}\right)-\mathbb{E}\left(L_{n}\right)-\frac{1}{2} \log n\right)=-C=-0.67165 \ldots
$$

This phenomenon suggests a question related to the shape of a random Ferrers diagram of $n$. It is interesting to determine how far from the largest block area 
the $L_{n} M_{n}$-area is if all block areas of the Ferrers diagram are arranged in nonincreasing order. This kind of rearrangement of blocks of the Ferrers diagram was studied by Fristedt [7, p. 710], who obtained a general limit theorem for the $r$ th block area. To state the problem in more precise way, we denote by $X_{n}^{(k)}$ the multiplicity of part $k(k=1, \ldots, n)$ in a random integer partition of $n$. Let $Z_{n}^{(r)}$ be the $r$ th largest member of the sequence $\left\{k X_{n}^{(k)}\right\}_{k=1}^{n}$. Erdös and Szalay [6] showed that

$$
\lim _{n \rightarrow \infty} \mathbb{P}\left(\frac{c}{\sqrt{n}} Z_{n}^{(1)}-\frac{1}{2} \log \frac{n}{c^{2}}-\log \log \log n \leq u\right)=e^{-e^{-u}}, \quad-\infty<u<\infty .
$$

Fristedt [7, Theorem 2.7] generalized this result to $Z_{n}^{(r)}$, where $r \geq 1$ is fixed. He showed that

$$
\begin{aligned}
& \lim _{n \rightarrow \infty} \mathbb{P}\left(\frac{c}{\sqrt{n}} Z_{n}^{(r)}-\frac{1}{2} \log \frac{n}{c^{2}}-\log \log \log n \leq u\right) \\
& =\int_{-\infty}^{u} \frac{\exp \left(-e^{-w}-r w\right)}{(r-1) !} d w, \quad-\infty<u<\infty .
\end{aligned}
$$

Let $R_{n}$ be the smallest value of $r$ such that $Z_{n}^{(r)}=L_{n} M_{n}$. We conjecture that

$$
\mathbb{E}\left(R_{n}\right) \asymp \log \log n, \quad n \rightarrow \infty
$$

This claim is supported by the following heuristic but non-rigorous argument. From the result of Theorem 2 it follows that

$$
\mathbb{E}\left(L_{n} M_{n}\right)=\frac{\sqrt{n}}{2 c} \log n+O(\sqrt{n}), \quad n \rightarrow \infty .
$$

In addition, a calculation of the expectation of the distribution in the right-hand side of (48) given by Fristedt [7, p. 708] could be used to show that

$$
\mathbb{E}\left(Z_{n}^{(r)}\right)=\frac{\sqrt{n}}{2 c}(\log n+2 \log \log \log n-2 \log r)+O(\sqrt{n}),
$$

if $r=r(n) \rightarrow \infty$ as $n \rightarrow \infty$. Combining (50) with (51), one may conclude that $\log r(n) \approx \log \log \log n$, which supports the claim in (49). We hope to return to this question in a future study.

\section{References}

[1] Abramovitz, M., Stegun, I.A.: Handbook of Mathematical Functions with Formulas, Graphs and Mathematical Tables. Dover Publ., Inc. New York (1965)

[2] Andrews, G.E.: The Theory of Partitions. Encyclopedia Math. Appl. 2, Addison-Wesley, Reading, MA (1976) 
[3] Archibald, M., Blecher, A., Brennan, C., Knopfmacher, A., Mansour, T.: Partitions according to multiplicities and part sizes. Australas. J. Combin. 66, 104-119 (2016)

[4] Dwight, H.B.: Tables of Integrals and Other Mathematical Data, 4th ed. MacMillan, New York (1961)

[5] Erdös, P., Lehner, J.: The distribution of the number of summands in the partitions of a positive integer. Duke Math. J. 8, 335-345 (1941)

[6] Erdös, P., Szalay, M.: On the statistical theory of partitions. In: Topics in Classical Number Theory, vol. I (G. Halasz ed.). North-Holland, Amsterdam, pp.397-450 (1984)

[7] Fristedt, B.: The structure of random partitions of large integers. Trans. Amer. Math. Soc. 337, 703-735 (1993)

[8] Grabner, P., Knopfmacher, A.: Analysis of some new partition statistics. Ramanujan J. 12, 439-454 (2006)

[9] Grabner, P., Knopfmacher, A., Wagner, S.: A general asymptotic scheme for the analysis of partition statistics. Combin. Probab. Comput. 23, 1057$1086(2014)$

[10] Hardy, G.H., Ramanujan S D.: Asymptotic formulae in combinatory analysis. Proc. London Math. Soc. 17(2), 75-115 (1918)

[11] Husimi, K.: Partitio numerium as occurring in a problem of nuclear physics. Proc. Phys.-Math. Soc. Japan 20, 912-925 (1938)

[12] Kessler, I., Livingston, M.: The expected number of parts in a partition of $n$. Monatsh. Math. 81, 203-212 (1976)

[13] Rademacher, H.: On the partition function $p(n)$. Proc. London Math, Soc. 43, 241-254 (1937)

[14] Richmond, L.B.: The moments of partitions, I. Acta Arith. 211, 345-373 (1912)

[15] Sloane, N.: On-line Encyclopedia of Integer Sequences (https://oeis.org/) 